\title{
Ultrasound monitoring of applied forcing, material ageing, and catastrophic yield of crustal structures
}

\author{
G. P. Gregori ${ }^{1,2}$, M. Lupieri ${ }^{1,2}$, G. Paparo ${ }^{1,3}$, M. Poscolieri ${ }^{1,2}$, G. Ventrice ${ }^{4}$, and A. Zanini $^{5}$ \\ ${ }^{1}$ Istituto di Acustica “O.M. Corbino”, C.N.R., Via Fosso del Cavaliere 100, 0133 Rome, Italy \\ ${ }^{2}$ ICES - International Centre for Earth Sciences, Via Fosso del Cavaliere 100, 00133 Rome, Italy \\ ${ }^{3}$ Italian Embassy, Buenos Aires, Argentina \\ ${ }^{4}$ P.M.E. Engineering, Rome, Italy \\ ${ }^{5}$ Alitalia, Power Plant Engineering Dept., Rome, Italy
}

Received: 20 July 2007 - Revised: 5 November 2007 - Accepted: 5 November 2007 - Published: 28 November 2007

\begin{abstract}
A new kind of data analysis is discussed - and a few case histories of actual application are presented concerning the physical information attainable by acoustic emission $(A E)$ records in geodynamically active or volcanic areas. The previous analyses of such same kind of observations were reported in several papers appeared in the last few years, and here briefly recalled. They are concerned with the inference of the forcing (" $F$ ") acting on the physical system, and on the ageing (" $T$ ") or fatigue of its "solid" structures. The new analysis here discussed deals with the distinction between a state of applied stress ("hammer regime"), compared to state of "recovery regime" of the system while it seeks a new equilibrium state after having been perturbed. For instance, in the case of a seismic event - and according to some kind of almost intuitive argument - the "hammer regime" is the phenomenon leading to the main shock, while the "recovery regime" deals with the well known aftershocks. Such same intuitive inference, however, can be investigated by a much more formal algorithm, aimed at envisaging the minor changes of the behaviour of the system, during its history and during its present dynamic evolution. As a demonstrative application, detailed consideration is given of $A E$ records - each one lasting for a few years - collected on the Italian peninsula vs. records collected on the Kefallinìa Island (western Greece). Such two areas are well known being characterised by some great comparative difference in their respective tectonic setting. When considering planetary scale phenomena, they appear comparatively very close to each other. Hence, they are likely being presumably affected by similar large-scale external actions, although they ought to be expected to respond in some completely different way. Such facts are clearly manifested by some substantially different $A E$ responses of the local crustal structures. However, a full understanding of such entire set of geodynamic
\end{abstract}

Correspondence to: M. Poscolieri

(maurizio.poscolieri@idac.rm.cnr.it) and tectonic details ought to require several year data series of $A E$ records, and/or (maybe) also simultaneous $A E$ records collected within some suitable array of $A E$ stations. Such understanding ought to permit the inference of the spatial features of the crustal stress propagation - including its diagnosis and "forecasting" - in addition to the temporal diagnosis and "prevision" that can be attained by isolated point-like $A E$ recording stations. Additional analyses are in progress.

\section{Introduction}

It is shown that forcing, ageing, and catastrophic yield of crustal solid structures can be effectively monitored and diagnosed by passive recording of acoustic emission $(A E)$ that, concerning the present applications, are focused on $H F A E$ (either $200 \mathrm{kHz}$ or $150 \mathrm{kHz}$ ) and $L F A E(25 \mathrm{kHz})$. The present paper - compared to a series of several previous papers - reports about a few new achievements. No previous details are here repeated. The interested reader ought to refer to such sources for more specific information (see References).

Three kinds of information can be inferred from either $H F$ $A E$ or $L F A E$. Tout court let us call them (i) $F$ for "Forcing"; (ii) $H$ for "Hammer" effect; and (iii) $T$ for "Time" or "ageing", which can be further distinguished into a pathological vs. physiological effect. Previous papers were mostly concerned with $F$ and $T$, and every paper is methodologically exhaustive for its respective concern. A synthetic methodological review of such entire set of items is given by Gregori and Paparo (2004).

The original target of the present study deals with $H$. Concerning $F$ and $T$, a short summary is here given as a premise for the subsequent discussion. Upon considering the comments of several colleagues, a basic premise appears worthwhile. Present engineering and Earth's sciences largely rely on differential calculus, and on the dynamics of rigid

Published by Copernicus Publications on behalf of the European Geosciences Union. 
(a)

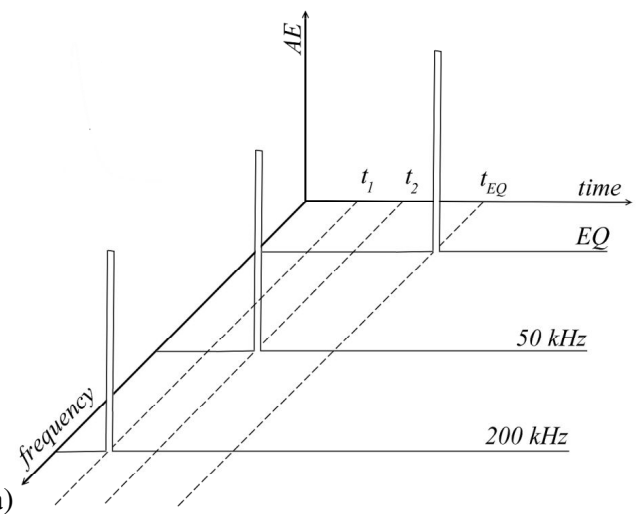

(b)

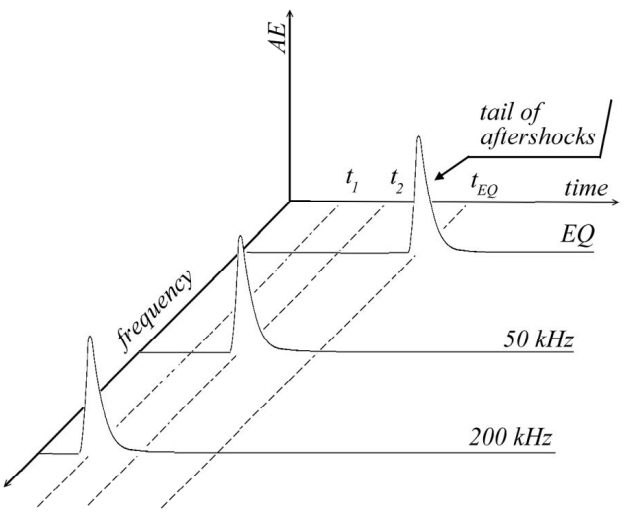

(c)

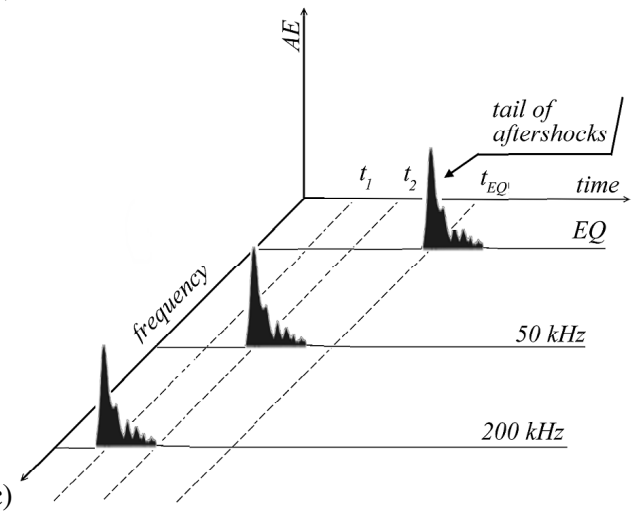

Fig. 1. (a) The bulk of the frequency of the released $A E$ decreases vs. time, and, as a first order approximation, the phenomenon can be depicted in terms of Dirac $\delta$-functions. (b) Upon closer physical consideration, every $\delta$-function ought to be substituted by a lognormal distribution. (c) An eventual externally applied additional effect (such as e.g. tidal modulation) sometimes results into an apparent trend looking like a damped oscillation. See text. Figure after Paparo and Gregori (2003) and Gregori and Paparo (2004). EQ means earthquake. During the rising phase of the lognormal distribution the physical system is in "hammer regime" (see Sect. 5), while during the tail it is in "recovery phase".

and continuous bodies, and infinitesimal calculus does not hold in the quantum domain. Even the Maxwell's laws are "thermodynamic" expressions of phenomena that ought to be more properly described by means of photons and Feyn- man graphs. In the same way, the kinetic theory of gases is a more detailed description of the laws of thermodynamics. But the usual model based on a "fog of billiard balls" cannot give justice for phase transitions, even with the refinement of the van der Waal's equation. Consider that PC and portable telephones had not be exploited by means of the Maxwell's laws alone, and quantum mechanics and solid state physics were essential. Similarly, $A E$ (ultrasounds) are often used by engineers for investigating the vibrations of structures, also referring to accelerometers, etc. But such approach in no way can be compared with the concern of the present paper, which is rather focused on the prime response of the atomic and molecular structures for diagnosing the very beginning of the ageing of materials, which testify a reduction of performance of the solid materials.

\section{Preliminaries}

\subsection{The $A E$ source}

An $A E$ source is associated with the release of energy originated by the yield of some chemical bonds within some crystal structure. The reaction chain concept applies, because one additional chemical bond is more likely (eventually) to yield close to the point where the mechanical strength of the solid object is comparatively weaker, due to the presence of some previous flaw. It is the principle idea justifying the cleavage plane of a crystal fracture.

Differently stated, an $A E$ signal can propagate through an ideal perfect elastic structure - i.e. through an ideal "solid" or "rigid" body. But it can release no $A E$, as the potential energy of every elastic bond is periodically transformed into kinetic energy - and viceversa. In contrast, the $A E$ release occurs only whenever a bond yields and some energy becomes available for propagating a newly generated $A E$.

The opposite extreme - compared to an ideal "elastic" structure - refers to the case history of an ideal "Newtonian fluid", where every displacement of some part of the system is always strictly proportional to the applied stress. This is the ideal "plastic" behaviour. Every such ideal body eventually transports $A E$, although by causing a damping, depending on the internal friction of the fluid.

Every physically existing body shall never be either an ideal solid or an ideal fluid. It is up to the researcher who (according to Einstein and others) must seek "simple" or "beautiful" models for her/his interpretation - deciding whether the physical system of her/his concern can fit either one such simplifying extrapolation to the case history of an "ideal" body. In general, one finds that only one such model is suited, provided that suitable account is given for the implicit approximations that constrain the reliability of the model within some given physical boundaries. 


\subsection{The time sequence of the $A E$ release}

The first yielding bonds are associated with geometrical sizes typical of the most elementary crystal structure. Owing to the aforementioned reaction-chain mechanism, the newly yielding bonds produce a coalescence - of some former flaws of comparatively smaller size - into larger size flaws. Hence, the typical geometrical size of the flaws increases with time, and the frequency of the released $A E$ consequently decreases. The principle is the same as the sound emitted by a violin cord when the geometrical length of the cord is progressively increased.

Therefore, the bulk of the frequency of the observed $A E$ decreases vs. time (Fig. 1; Paparo and Gregori, 2003). As a first order approximation, the phenomenon can be depicted in terms of Dirac $\delta$-functions, where every such $\delta$ function is centred on some given $A E$ frequency. However, upon closer physical consideration - i.e. upon considering that flaws of some given comparatively smaller size are still evolving while the population of some larger size flaws is already in progress - every such $\delta$-function ought to be substituted by a lognormal distribution. In addition, an eventual externally applied additional effect (such as e.g. a tidal modulation) sometimes results into displaying some apparent trend looking like a damped oscillation. Such effect was observed e.g. either in volcanic $A E$ records (Vesuvius), or in $A E$ records in a tectonically active area (the Raponi site). For both such examples refer to Paparo and Gregori (2003).

In the case of a seismic event, the $A E$ are observed first at comparatively higher frequency, i.e. the $H F A E$. Then, progressively lower frequency $A E$ (i.e. $L F A E$ ) are observed. Then, the seismic roar is listened, and later on the mechanical vibrations are detected by accelerometers, or by some specific mechanical structures within buildings, etc. Finally, whenever the $A E$ frequency is decreased down to $\sim 0.5 \div 1 \mathrm{~Hz}$, the destructive shock occurs.

The aforementioned oscillation of the tail of the lognormal distribution, which is characteristic of the $A E$ events, is similar to - though physically different compared to - the well known time series of earthquakes that is called of "aftershocks". In fact, it has to be stressed that the aftershocks of an earthquake are caused by a different mechanism. Consider e.g. a bar that is subjected to an external mechanical action, until it breaks into two parts. Such parts are subjected to the same action, and are further broken, each one into two smaller bars, etc. Every such event generates a shock, resulting into a time series of events, which in general are of decreasing intensity, etc. This is the rationale for the generation of earthquakes, which is much different compared to the aforementioned $A E$ case history, where the mechanism rather appeals to the timing of the coalescence of flaws associated with the generation of $A E$ of decreasing frequency. The final result is apparently the same, although the physical timing relies on a different physical justification.

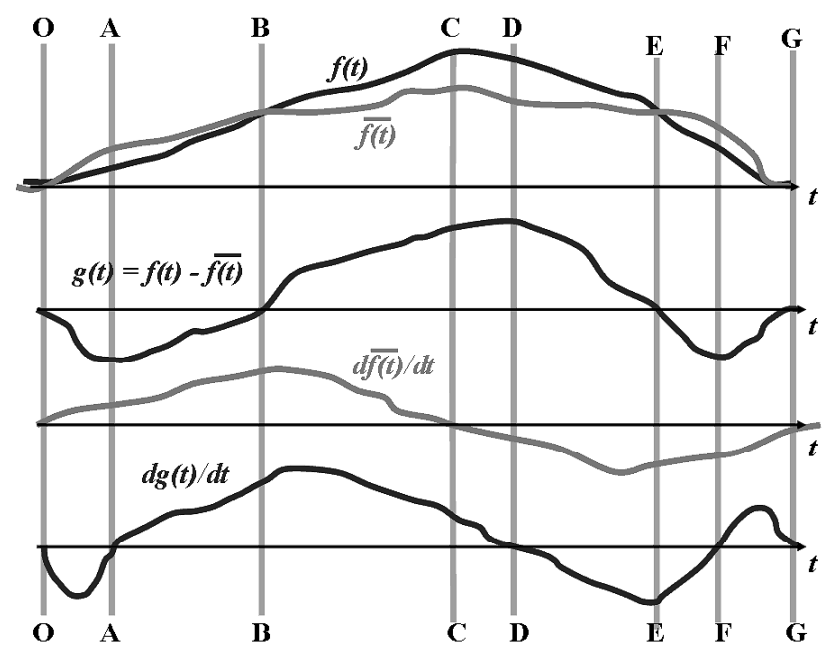

Fig. 2. Qualitative cartoon showing an arbitrary observational datum $f(t)$, with its smoothing average over a suitable time interval, and its residual, and their respective time derivatives. See text.

In general, therefore, it has to be expected that the $H F$ $A E$ are representative of the comparatively earlier stages of the evolution of the system. For instance, in the case of the crustal stress, the release of $H F A E$ reflects the former externally applied tectonic action. In contrast, the comparatively lower frequency $A E$, i.e. the $L F A E$, do reveal some processes or phenomena that occur within the physical system when its original ideal "solid" crystal structure already suffered by some relevant ageing, or deterioration. Therefore, in the case of crustal stress, it has to be expected that the $L F A E$ records appear more correlated with tectonic agents, compared to the $H F A E$ that respond rather to some former trigger, occurring when the crystal structure was still almost perfectly shaped. In such same respect, it ought to be pointed out that the information provided by accelerometers or by seismometers deals with a much evolved stage of the evolution of the system, and it applies when a "thermodynamic" description can be significantly applied in terms of continuous functions, differential calculus, and of vibrations of structures. However, such much later stage of the phenomenon cannot be representative of the original crustal stress propagation that was the real precursor of the processes that originally triggered the mechanism that finally led to the catastrophic yield.

As a general rule - while dealing with every specific actual application - both $H F A E$ and $L F A E$ ought to be monitored, and their respective inferences suitably analysed. In fact, on some occasions either one $A E$ or the other results comparatively more effective, depending on the kind of phenomenon that results more sensible concerning the final effect to be monitored or forecasted. In addition, consider that - on a few case histories here reported and dealing with some early investigations on $A E$ - only one $A E$ frequency was recorded. 

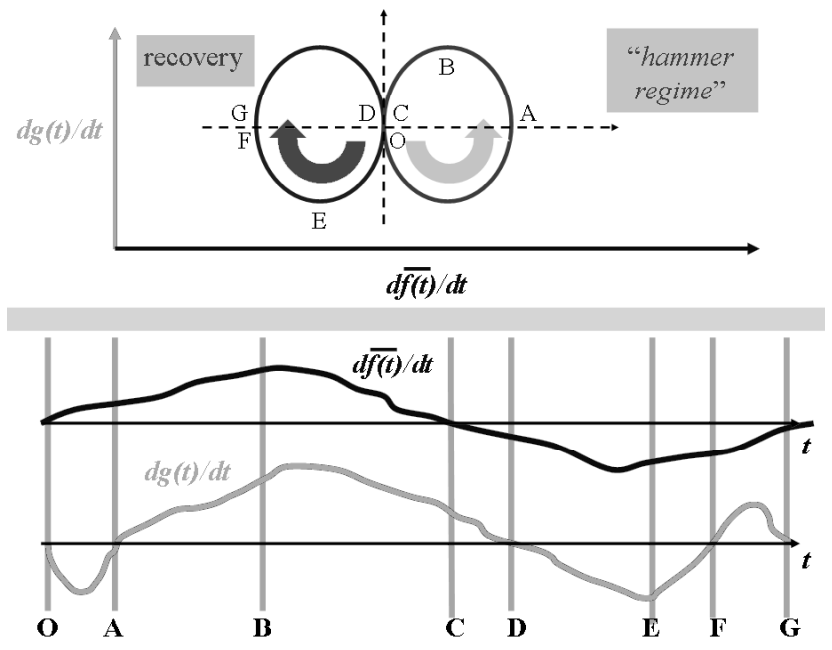

Fig. 3. Qualitative cartoon, showing how to infer the "hammer regime" vs. the "recovery regime"

\subsection{The $A E$ probe}

It should be pointed out that an $A E$ recording device is composed of the sensor set with amplifier, etc. and data logger, plus in addition some whole part of the same physical system being monitored. In fact, if the $A E$ sensor (i.e. the acoustic transducer) monitors e.g. some solid outcrop (granite, limestone, lava, etc.), it records the $A E$ released within such entire (approximately) "solid" object up to some large distance from the transducer itself. Such distance cannot be envisaged a priori, as it depends on two crucial physical factors: the intensity of the prime $A E$ source, and the damping of the signal, while it propagates from its source through the transducer.

In any case, the entire composite recording device, composed of the transducer plus the entire "solid" object - up to some suitable and unknown distance from the transducer has to be considered as a unique probe. Such fact has important consequences. For instance, while monitoring a small lava outcrop (e.g. a dyke) on the flanks of Vesuvius, indeed we do monitor a large fraction of the entire volume of its volcanic edifice, due to the (unknown) underground extension of the natural probe represented by some kind of elongated "tongue" of solidified lava.

Under such favourable circumstances, the large size of the probe - and its effectiveness in transporting $A E$ - do imply an excellent signal-to-noise ratio in the final records.

\subsection{The prime cause for the $A E$ release}

Distinction ought to be made between an "external" vs. an "internal" forcing as the prime cause that triggers the $A E$ release.

The "external" forcing can be either exogenous or endogenous. For instance, a tectonic stress resulting from a geodynamic action results into a stress applied to some solid

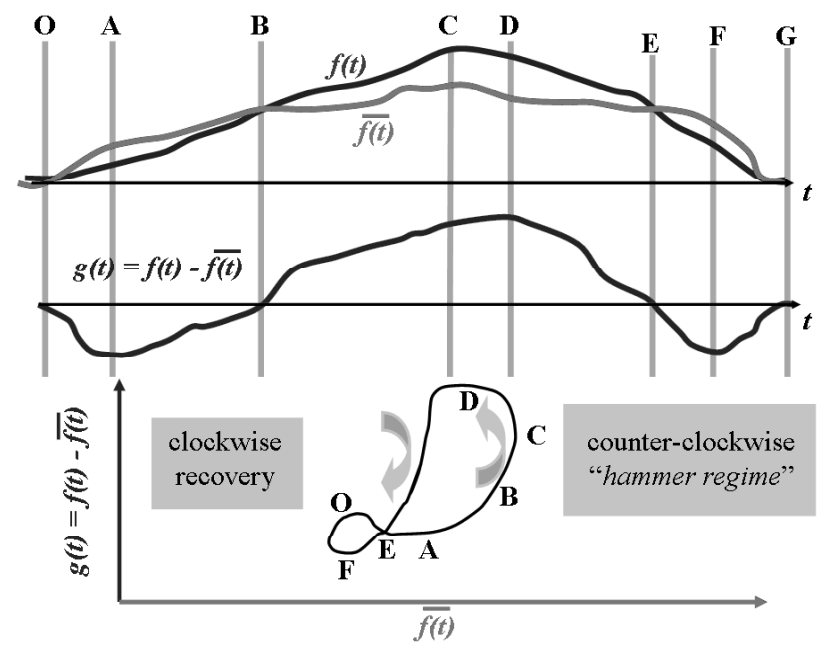

Fig. 4. Qualitative cartoon, showing the "hammer plot" for inferring "hammer regime" vs. "recovery regime".

portion of the crust, causing the yield of some of its crystal bonds: it is an exogenous trigger. In contrast, an endogenous action or trigger is typically associated e.g. with the diffusion, at some high pressure, of some hot fluid through the pores of the "solid" body. Such pressure stress originates crystal bond yielding and $A E$ release. That is, even an endogenous trigger is a cause that operates "externally" with respect to the crustal structure of concern.

The "internal" forcing is rather concerned with the physiological evolution of the system, after having suffered by some external action. That is, the physical system - after having been subject to some "external" action - goes through some recovery state, and - while spending such transient state during which it seeks some new equilibrium configuration - it eventually releases $A E$. This is an "internal" trigger, as no external action is implied, either by some mechanical stress, or by some endogenous fluids or other: it is just a natural evolution of the system, after having suffered by some "externally" applied paroxysm.

\section{5 $A E$ transmission vs. teleconnection}

Some relevant physical concern deals with the maximum distance at which an $A E$ release can be detected - and such concern was the likely prime cause of the limited amount of previous investigation carried out in the natural environment by means of $A E$. In fact, as a standard, the $A E$ damp off within some very short distance through loose material, resulting practically useless for any monitoring purpose.

Hence, whenever $A E$ records are monitored at sites located at $\sim$ several $100 \mathrm{~km}$ from their likely source - and such correlation appears observationally unquestionable - such observational fact can be explained either by $A E$ transmission or by $A E$ teleconnection. 
$A E$ transmission ought to require the existence of some waveguide, such as some kind of "rigid", "solid", uninterrupted structure connecting $A E$ source and $A E$ recording site. Such physical condition, however, is likely to occur in homogeneous bodies, such as within a man-made machinery, or in concrete buildings or constructions, etc., while it is unlikely to occur in natural structures - except at most under very exceptional circumstances.

$A E$ teleconnection rather implies that a common cause triggers the $A E$ prime action (exogenous or endogenous), which is responsible for the $A E$ release that is recorded at different sites. $A E$ teleconnection typically occurs in natural structures (crustal slabs, volcanic edifices, etc.). For instance, according to clear observational inference, the entire Italian peninsula can be likened to one unique "solid" crustal slab, which is eventually stressed by some externally applied geodynamic action, by which crises of release of $A E$ are simultaneously monitored at sites even $\sim$ several hundred $\mathrm{km}$ far apart from each other.

\section{$3 \quad F \equiv$ Forcing}

The intensity of the $A E$ signal is larger for a comparatively more intense applied forcing, although in general such relation is not linear. Several such examples were found.

A diurnal (mostly thermoelastic, and partly tidal) effect was clearly recognised on a massif in the central Apennines (Gran Sasso). During day-time warming, the outer layers of rocks (limestone and dolomite) expand over the innermost rock volume, which is cooler and more contracted. In contrast, during night-time, the outer rock layers, while cooling, do contract outside the warmer and more expanded rocks. Hence, during night-time, i.e. during rock cooling, a greater amount of $A E$ release is monitored. The difference of such daily variation - when comparing different days - results from the different solar heating of the mountain depending on meteorological conditions. Refer to Gregori and Paparo (2004).

At the Raponi site (located in Orchi, hamlet of Foligno town, in central Italy), and in the Kefallinìa island (western Greece), an annual wave of crustal stress was clearly recognised. The present interpretation is in terms of a wave of crustal stress crossing the entire Greek and Italian areas, maybe of planetary scale and origin. Additional investigations are needed in order to assess the diagnostic potential of such unexpected and clear evidence. Refer to Paparo et al. (2006) and Poscolieri et al. (2006, 2006a) for additional details and discussion.

The $F$ information also resulted into a series of seemingly clear and significant earthquake precursors. Three such case histories ought to be recalled.

At the site Giuliano, close to Potenza (southern Italy), several geophysical monitoring devices were in operation during the days that preceded the earthquake that occurred on 3

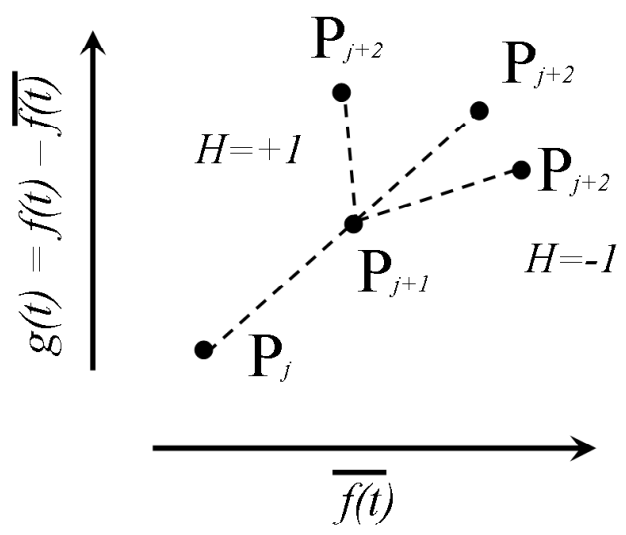

Fig. 5. Qualitative cartoon, showing a three-point detail of a "hammer plot". Consider three consecutive points $\mathrm{P}_{j}, \mathrm{P}_{j+1}, \mathrm{P}_{j+2}$. Draw the line through $\mathrm{P}_{j}$ and $\mathrm{P}_{j+1}$. If $\mathrm{P}_{j+2}$ falls to the left of such line it is concluded that $\mathrm{P}_{j+2}$ is associated with a state of $\mathrm{H} \equiv 1$, if to the right of the line $\mathrm{H} \equiv-1$. If $\mathrm{P}_{j+2}$ falls right on such line, $\mathrm{P}_{j+2}$ is associated with a state of $\mathrm{H} \equiv 0$.

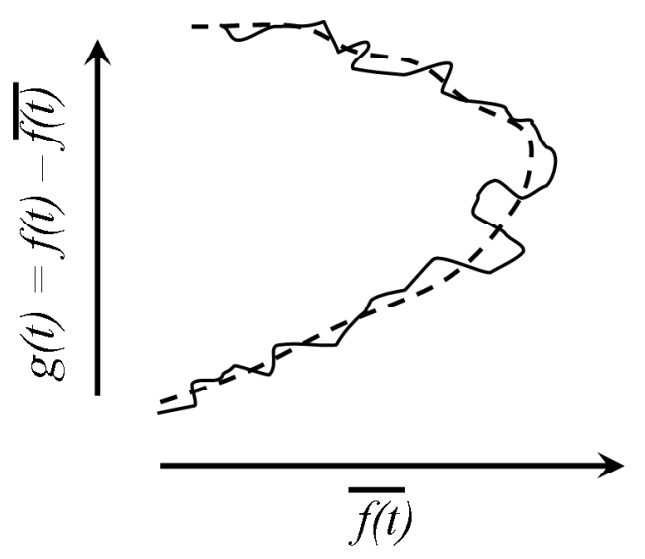

Fig. 6. Qualitative cartoon, showing a general trend of a point sequence on a "hammer plot". Suppose that the leading physical trend is suggestive of an counterclockwise behaviour (dashed line). Observational errors, however, produce a scatter of the plotted points (solid line). According to the procedure sketched in Fig. 5, the result shall appear like a series of both $H=+1$ and $H=-1$, with a prevailing component of $H=+1$ values. The concern is about envisaging a significant filter aimed at focusing in some objective and unquestionable way the leading physical trend, independent of the perturbation produced by the scatter originated by the observational errors.

April 1996, of magnitude $\mathrm{Md}=4.6$ with epicentre at $\sim 18 \mathrm{~km}$ from the $A E$ recording site. Some $\sim 2$ days before the shock, the $L F A E$ intensity went out of scale for a time lag of $\sim 1$ day. The existence of a teleconnection mechanisms over $\sim 18 \mathrm{~km}$ distance is an observational fact, although it is essentially unexplained.

On the Italian peninsula, the $H F A E$ intensity - recorded at several $100 \mathrm{~km}$ from the epicentre - displays a violent crisis 


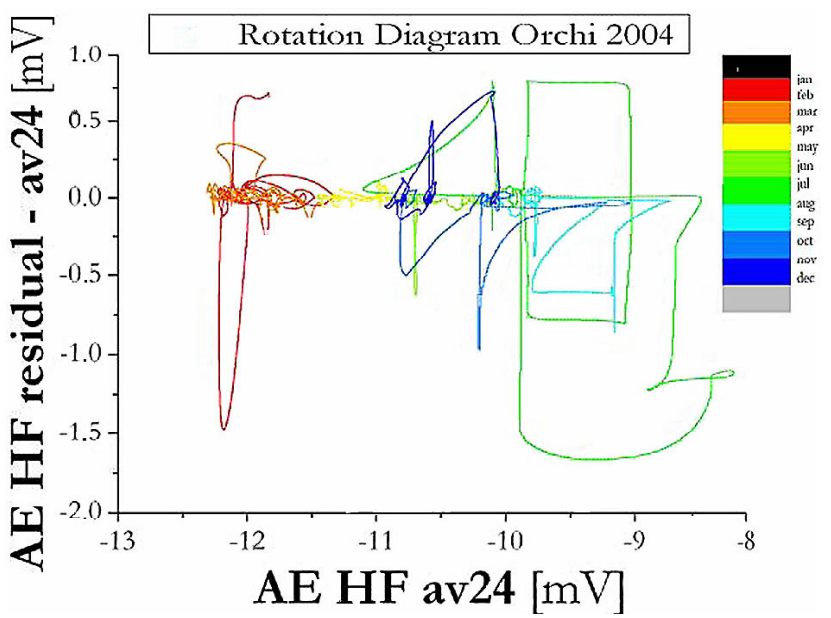

Fig. 7. $H F A E$ "hammer plot" for the Raponi site during 2004.

some $\sim 7 \div 8$ months before the shock, and the $L F A E$ some $\sim 2$ months before the shock. Such inference was checked on a few occasions. Refer to Paparo et al. (2006) for details.

Concerning the Kefallinìa Island (western Greece) - in addition to the aforementioned $H F A E$ annual wave - a stress soliton was recorded (mainly in the $L F A E$ ), presumably crossing the entire area and lasting several months. The assessment and recognition of actual earthquake precursors, however, need for some much longer series of records. In any case, the much different tectonic setting - compared to the Italian peninsula - justifies an expectedly much different behaviour. Refer to Poscolieri et al. (2006) for details. Concerning $A E$ records in volcanic areas, the volcano Peteroa (Argentina, in the Planchon volcanic complex) displays regular bursts of $H F A E$ that envisage a clear tidal control. The volcanic edifice operates like the valve of a pressure cooker: whenever the ebb-and-flow of the tide lifts the weigh of the valve, a burst of endogenous hot fluids - which are no more constrained by the valve - produces a peak of $A E$ release (Ruzzante et al., $2007^{1}$ ). That is, the volcano Peteroa appears to respond with a surprisingly great precision to the tidal trigger.

\section{$4 \quad T \equiv$ Time or "ageing"}

The focus is on the fatigue of the material. It is some kind of ultrasonic yelp, or whine, or moan, or whimper, which is independent of the intensity of the applied forcing, and it is only related to the ageing of the material. That is, the intensity $F$ of the $A E$ signal is related to the strength of the applied stress (Sect. 3). But the timing of the $A E$ release re-

\footnotetext{
${ }^{1}$ Ruzzante, J., Lòpez Pumarega, M. I., Piotrowski, R., Gregori, G. P., Paparo, G., Poscolieri, M. and Zanini, A.: Acoustic emission $(A E)$, tides and degassing on the Peteroa volcano (Argentina), in preparation, 2007
}

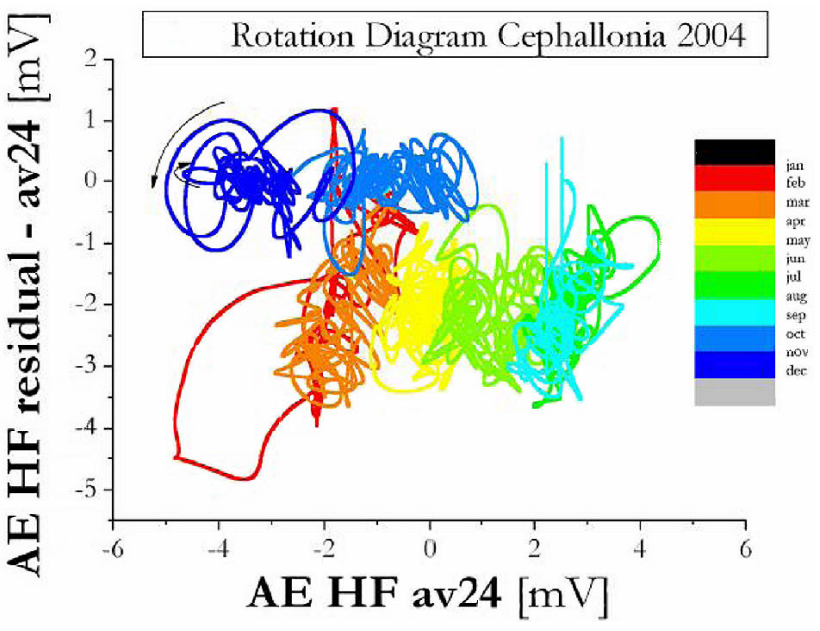

Fig. 8. $H F A E$ "hammer plot" for the Kefallinìa island during 2004.

flects the evolutionary stage of the reaction chain process (see Sect. 2.1). Such information is clearly inferred by applying a fractal analysis to the recorded $A E$ signal. $^{2}$

The Giuliano (Potenza) case history (Sect. 3) showed a progressive decrease - lasting for at least 2 months - from an $L F A E$ fractal dimension $D_{t} \sim 1$ (equivalent to full nonorganization of the fracture surface) to $D_{t} \sim 0.45 \div 0.4$ (the null value for $D_{t}$ corresponds to perfect organization of the fracture into a cleavage plane). The records were collected at Giuliano, i.e., at $\sim 18 \mathrm{~km}$ distance from the epicentre. But the same inference resulted to apply also for the Colfiorito earthquake, which had epicentre much farther away. However, concerning the records at the Raponi site - i.e. collected right at the epicentre of the Colfiorito earthquake - the $L F A E$ fractal dimension $D_{t}$, showed no equivalent clear evidence referring to the Molise earthquake. The earthquake precursor was very clear in the $F$ effect (for both $H F A E$ and $L F$ $A E$ ), though it was not evident in the $L F A E$ fractal dimension $D_{t}$. The problem requires harder thinking on a wealthier database. See Paparo et al. (2006).

Concerning the Kefallinìa case history, both $H F A E$ and $L F A E$ fractal dimensions $D_{t}$ could be evaluated during some exceedingly short time lag for getting any relevant useful inference - upon considering the particularly complicated tectonic setting of the area. See Poscolieri et al. (2006).

The $A E$ records in volcanic areas (Vesuvius and Stromboli) resulted particularly effective.

\footnotetext{
2 The original signal is first smoothed by a weighted running average over 24 hours time interval, in order to get rid of all diurnal effects (thermoelastic and/or tidal). The residual - between original record and smoothed average - is investigated by selecting a series of relative maxima above some suitable threshold. The time series of such relative maxima -i.e. a so-called point-like process - is then analysed by fractal analysis.
} 
In the Vesuvius case, the $L F A E D_{t}$ resulted much effective (Paparo et al., 2004). Whenever the endogenous pressure pushes the hot fluids into the pores of the solid parts of the volcanic edifice, the crystal bonds yield in some random way - deriving from a process of 3-D diffusion - and it is found $D_{t} \sim 1$. In contrast, when the endogenous pressure of the hot fluids has a temporary decrease, the volcanic edifice being no more supported by the endogenous pressure - can temporarily experience several micro-collapses, and the released $A E$ displays $D_{t}<1$. In this way, it is possible to assess - using tout court an intuitive though expressive term - when Vesuvius is "inflating", compared to the times when it is "deflating". The effect is clear in $L F A E$, while it is less clear for $H F A E$ (see Sect. 2.2).

It should be pointed out that such interpretation is further supported by the aforementioned evidence got about the volcano Peteroa (Sect. 3).

In addition, concerning seismic activity on Vesuvius - and consistently with the expectation of such interpretation - during "inflation" time a conspicuous seismic activity is observed, although resulting into a series of a large number of instrumental shocks. In contrast, during "deflation" time the activity is concentrated in a few shocks of larger intensity. The integral of the total energy release during "inflation" is much larger than the integral during "deflation".

Concerning Stromboli, the $H F A E D_{t}$ gave a much clear precursor - with an advance of at least 5 months - of the crisis that occurred at the end of 2002 (see Gregori and Paparo, 2006). It should be pointed out that the $F$ of $H F A E$ gives a completely different information compared to the fractal dimension $D_{t}$, thus confirming the (expected) fact that different physical parameters give different inferences.

\section{$5 \quad H=$ "Hammer" effect}

Every phenomenon can be distinguished into two opposed physical states, i.e. whether it is subject to some forcing, or in contrast whether it is rather in a stage of recovery. Differently stated, such distinction depends one whether the $A E$ release occurs due to an active external forcing, or rather due to its "aftershock" recovery. Let us briefly call such distinction the "hammer effect", envisaging that - during forcing the system is like being hit by a hammer, while - during recovery - it seeks a new equilibrium after the hammer stroke. "Hammer time" will be conventionally defined by an index $H \equiv 1$, and "recovery time" by an index $H \equiv-1$, while even the indeterminate case history $H \equiv 0$ will be eventually considered.

Such general principle - and the evaluation of $H$ - can be operatively applied as follows. For clarity purposes, refer to the qualitative cartoon of Fig. 2.

Let some given (arbitrary) physical measurement being represented by a function $f(t)$. Call $\bar{f}(t)$ some moving average over a suitable time lag (computed accordingly, and be-

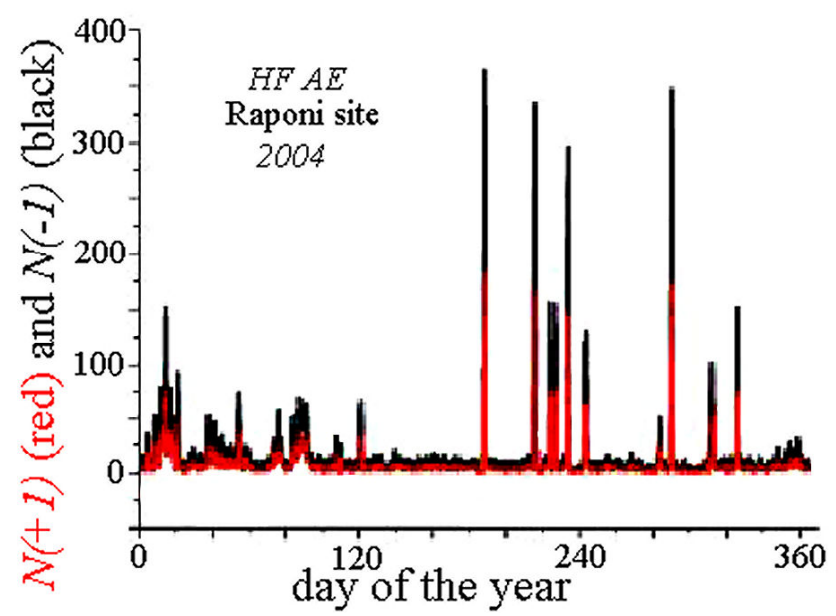

Fig. 9. Total number of $H=+1$ (red) and $H=-1$ (black) computed per day for $H F A E$ at the Raponi site during 2004.

ing either weighted or not). Call $g(t)=f(t)-\bar{f}(t)$ the residual. Then, suppose to plot $d \bar{f}(t) / d t$ and $d g(t) / d t$ vs. $t$.

Plot (Fig. 3) $d g(t) / d t$ vs. $d \bar{f}(t) / d t$ and - by a little thinking - promptly realise that the "hammer regime" corresponds to a counterclockwise trend over such plot. In contrast, a "recovery regime" corresponds to a clockwise rotation.

The same result is attained by plotting (Fig. 4) $g(t)=f(t)-\bar{f}(t)$ vs. $\bar{f}(t)$, which will be briefly called "hammer plot".

If one applies such "hammer" analysis to an ideal lognormal trend - such as the one qualitatively represented in Fig. 1 - it is found that the smaller is the radius of the loop on the "hammer plot" the sharper is the lognormal distribution. Or the width of the lognormal distribution determines the size of the loop in the hammer diagram. Therefore, plotting the loop permits recognising, almost in real time, when a structure is subject to slow or fast deformations (such as during fault slippage etc.).

Practical application of the "hammer plot" is carried out as follows. Consider three consecutive points $\mathrm{P}_{j}, \mathrm{P}_{j+1}, \mathrm{P}_{j+2}$ plotted on the "hammer plot" (Fig. 5). Draw the line through $\mathrm{P}_{j}$ and $\mathrm{P}_{j+1}$. If $\mathrm{P}_{j+2}$ falls to the left of such line state that $\mathrm{P}_{j+2}$ is associated with a state of $H \equiv 1$, if to the right of the line $H \equiv-1$. If $\mathrm{P}_{j+2}$ falls right on such line state that $\mathrm{P}_{j+2}$ is associated with a state of $H \equiv 0$.

A crucial point is concerned with the role of errors. If the physical system is experiencing e.g. a "hammer state", its "hammer plot" shall result counterclockwise (Fig. 6). The plotted points, however, are subject to the observational errors, which shall produce a scatter of the plotted points around the leading counterclockwise trends. Therefore, the sequence of computed $H$ values shall contain some large number of $H \equiv-1$ randomly included into some comparatively larger ensemble of $H \equiv 1$. 


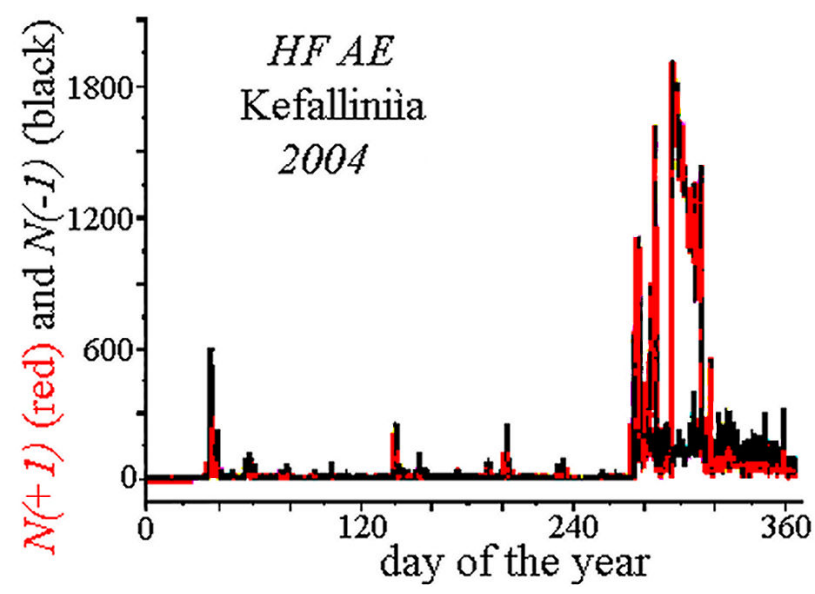

Fig. 10. Total number of $H=+1$ (red) and $H=-1$ (black) computed per day for $H F A E$ at the Kefallinìa Island during 2004.

The concept is better explained in terms of an intuitive - though, strictly speaking, not rigorous - analogy. Consider the trajectory of a molecule within the stream of a fluid, which shows a Brownian motion along its average flow line. The track of points drawn in the "hammer plot" shows some scatter equivalent to such Brownian motion, clustered around a main flow line, which is the information of physical concern for investigating the hammer effect. In the ultimate analysis, natural phenomena occur independent of the way they are observed and described. The hammer effect analysis is just an expressive way of representing some parameter - derived by a suitable analysis of observations - that ought to help in focusing on some leading and large scale characteristics of the evolution of the physical system.

Therefore, every computed series of $H$ values must be suitably treated statistically, in order to smooth out the devastating role of the scatter caused by the observational errors. Such algorithms shall be assessed after suitable harder thinking, and several concrete checks-and-trial applications to different case histories. Only very few such applications are here presented, while a systematic re-handling is currently in progress of all $A E$ data series available to the authors.

Figure 7 shows the "hammer plot" of the $H F A E$ monitored at the Raponi site during 2004. Apart some lesser oscillations, there is some approximate seasonal trend from the left (February) through June (extreme right) returning halfway at the centre of the plot in December. But no interpretation seems as yet possible, being the likely consequence of a change in the "external" tectonic actions that are applied to the area.

Much different appears the $H F A E$ "hammer plot" for 2004 in the Kefallinìa island (Fig. 8). The difference between Figs. 7 and 8 are certainly concerned with the respective tectonic settings of the two regions.

Such difference can be further inspected by counting the total number of $H=+1$ (red) and $H=-1$ (black), at the
Raponi site (Fig. 9) and at Kefallinìa (Fig. 10). At the Raponi site, hammer and recovery regimes appear correlated and varying synchronously. In contrast, at Kefallinìa the tectonic setting implies close and repeated "hammer" strokes, mainly during October $\div$ November.

The physical interpretation of such different inferences shall require harder thinking, and simultaneous consideration of the $F, H, T$ effects associated with both $H F A E$ and $L F$ $A E$, including their apparent correlation with the seismic activity within some suitably large area around the $A E$ recording site (in progress).

No simple intuitive model seems as yet possible, and a full understanding of phenomena could require an array of simultaneous $A E$ recording stations, collected during some long time lag.

Some harder thinking is needed. The $F, H, T$ information got by $H F A E$ and $L F A E$ - recorded in the natural environment - envisages intriguing possibilities that, however, have to be physically interpreted by a cross-reference with different tectonic settings, and with different applied "external" geodynamic actions. Similar and/or analogous investigations are to be carried out in the laboratory, concerning all materials used in buildings or in machineries, in order to inspect the potential applications for security and/or for the recovery of ageing structures (e.g. Carpinteri et al., 2007). At present, it appears premature giving an account of such achievements.

Acknowledgements. The authors express their gratitude to A. Raponi and A. M. Raponi for kindly hosting the $A E$ monitoring apparatus in the basement of their house at Orchi (Italy) and to the Greek colleagues who have been collecting the $A E$ data in the Kefallinìa island. In addition, we thank I. Marson for several enlightening discussions, and for financial support in managing the Kefallinìa island measurements.

Edited by: P. F. Biagi

Reviewed by: two anonymous referees

\section{References}

Carpinteri, A., G. Lacidogna, and G. Niccolini,: Acoustic emission monitoring of medieval towers considered as sensitive earthquake receptors, Nat. Hazards Earth Syst. Sci., 7, 251-261, 2007, http://www.nat-hazards-earth-syst-sci.net/7/251/2007/.

Cello, G. and Malamud, B. D. (Eds): Fractal analysis for natural hazards, Geol. Soc. Lond., Special Publ., 261, 1-172, 2006.

Gregori, G. P. and Paparo, G.: The Stromboli crisis of $28 \div 30$ December 2002, Acta Geod. Geophys. Hung., 41(2), 273-287, 2006.

Gregori, G. P. and Paparo, G.: Acoustic emission (AE). A diagnostic tool for environmental sciences and for non destructive tests (with a potential application to gravitational antennas), in Schröder, 166-204, 2004.

Paparo, G., Gregori, G. P., Taloni, A., and Coppa, U.: Acoustic emissions $(A E)$ and the energy supply to Vesuvius - "Inflation" and "deflation" times, Acta Geod. Geophys. Hung., 40(4), 471480, 2004. 
Paparo, G. and Gregori, G. P.: Multifrequency acoustic emissions $(A E)$ for monitoring the time evolution of microprocesses within solids, Reviews of Quantitative Nondestructive Evaluation, 22, AIP Conference Proceedings, edited by: Thompson, D. O. and Chimenti, D. E., 1423-1430, 2003.

Paparo, G., Gregori, G. P., Poscolieri, M., Marson, I., Angelucci, F., and Glorioso, G.: Crustal stress crises and seismic activity in the Italian peninsula investigated by fractal analysis of acoustic emission $(A E)$, soil exhalation and seismic data, In Cello and Malamud, 47-61, 2006.

Poscolieri, M., Lagios, E. Gregori, G. P., Paparo, G., Sakkas, V. A., Parcharidis, I., Marson, I., Soukis, K., Vassilakis, E., Angelucci, F., and Vassilopoulou, S.: Crustal stress and seismic activity in the Ionian archipelago as inferred by combined satellite and ground based observations on the Kefallinìa Island (Greece), In Cello and Malamud, 63-78, 2006.
Poscolieri, M., Gregori, G. P., Paparo, G., and Zanini, A.: Crustal deformation and $A E$ monitoring: annual variation and stresssoliton propagation, Nat. Hazards Earth Syst. Sci., 6, 961-971, 2006a.

Schröder, W. (Ed.): Meteorological and geophysical fluid dynamics (A book to commemorate the centenary of the birth of Hans Ertel), Arbeitkreis Geschichte der Geophysik und Kosmische Physik, Wilfried Schröder/Science, Bremen, 417 pp., 2004. 